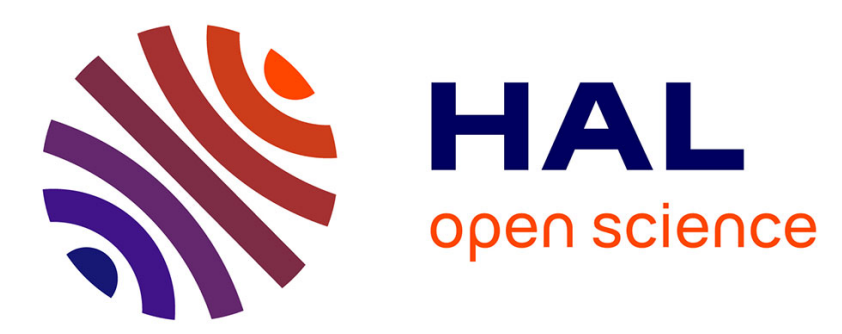

\title{
A theoretical investigation of the turbulent structures tilting measurements with radial correlation Doppler reflectometry
}

O L Krutkin, E. Gusakov, Stéphane Heuraux

\section{- To cite this version:}

O L Krutkin, E. Gusakov, Stéphane Heuraux. A theoretical investigation of the turbulent structures tilting measurements with radial correlation Doppler reflectometry. Plasma Physics and Controlled Fusion, 2020, 62, pp.045004. 10.1088/1361-6587/ab71f5 . hal-02434240

\section{HAL Id: hal-02434240 \\ https://hal.univ-lorraine.fr/hal-02434240}

Submitted on 9 Jan 2020

HAL is a multi-disciplinary open access archive for the deposit and dissemination of scientific research documents, whether they are published or not. The documents may come from teaching and research institutions in France or abroad, or from public or private research centers.
L'archive ouverte pluridisciplinaire HAL, est destinée au dépôt et à la diffusion de documents scientifiques de niveau recherche, publiés ou non, émanant des établissements d'enseignement et de recherche français ou étrangers, des laboratoires publics ou privés. 


\title{
A theoretical investigation of the turbulent structures tilting measurements with radial correlation Doppler reflectometry
}

\author{
O. L. Krutkin ${ }^{1,2}$, E. Z. Gusakov ${ }^{1}$, S. Heuraux ${ }^{2}$ \\ ${ }^{1}$ Ioffe Institute, St Petersburg, Russia \\ ${ }^{2}$ Institut Jean Lamour UMR 7198 CNRS, Univ. de Lorraine, BP 50840, 54011 Nancy, France
}

\begin{abstract}
A new technique for measuring the tilting of the turbulent structures is studied analytically within the framework of Born approximation. Probing with O-mode, linear density profile and slab geometry are considered. Analytical expressions are derived for scattering signal and crosscorrelation function of scattering signals valid for different probing frequencies. Comparison with previously used "intuitive model" is made, discrepancies pointed out and a more general expression for narrow probing beam is derived. General conclusions about the technique are made and a possible alternative is suggested. Analytical results are validated with linear numerical simulations employing reciprocity theorem, importance of poorly localized smallangle scattering is confirmed.
\end{abstract}

\section{Introduction}

One of the major physical problems arising in magnetic confinement fusion devices is the control of plasma turbulence and the anomalous transport associated with it. According to the present understanding, the turbulence, namely, drift wave turbulence is responsible for degradation of the plasma confinement. This makes it essential for confinement research to develop the diagnostic tools capable of measuring its parameters.

One of them is radial correlation Doppler backscattering, commonly referred to as radial correlation Doppler reflectometry (RCDR), proposed in [1] and used widely in experiment both in stellarators [2] and tokamaks [3, 4]. The diagnostics allows, under certain conditions to directly measure radial correlation function of the electron density perturbations. The diagnostic is based on probing the plasma volume with two microwave beams at oblique incidence with respect to the magnetic surface [1]. The probing is performed in the presence of a cut-off at close frequencies one of which is kept constant while the other one is varied. For each frequency difference the correlation of the scattered signals is measured. The resulting correlation function is then related to the spatial correlation of electron density fluctuations based on the cut-off separation for the probing frequencies [5].

While diagnostic benefits from the simplicity of execution, extensive theoretical [6-10] and numerical [11-13] analysis of the diagnostic have revealed that there is a number of factors that can complicate the relation between the correlation of the measured scattered signals and properties of density fluctuations. Such factors include nonlinear scattering effects $[8,9,12]$ as well as poorly localized small-angle scattering effects in the linear case $[5,7,12]$ or even geometrical effects [10]. These effects are well-described both in aforementioned works and in the papers dedicated to simpler diagnostic configurations [14-17]. Methods and criteria are suggested to allow for correct interpretation of the experimental data. 
One of the recent developments of the RCDR diagnostic is a technique suggested for indication of tilted turbulent structures rotating in poloidal direction [18] and for determination of their tilt angle [19]. This method, however, is based on an intuitive interpretation, ignoring the complexity of wave scattering process and consequently aforementioned effects. The goal of this work, therefore, is to consider this technique in the framework of a strict linear theory and to determine applicability and limits of the intuitive approach to data interpretation.

\section{Basic analytical equations}

Within this paper, plasma probing with the wave of the ordinary polarization (O-mode) is considered in the framework of the slab geometry. This allows us to obtain straightforward expressions while retaining the qualitative results. The background density profile is assumed to be linear along the radial coordinate and uniform along the poloidal one. The coordinates, named respectively as $x$ and $y$, while the z-direction is aligned with the applied magnetic field and not needed in this work. Geometry used in the consideration is illustrated on Fig. 1.

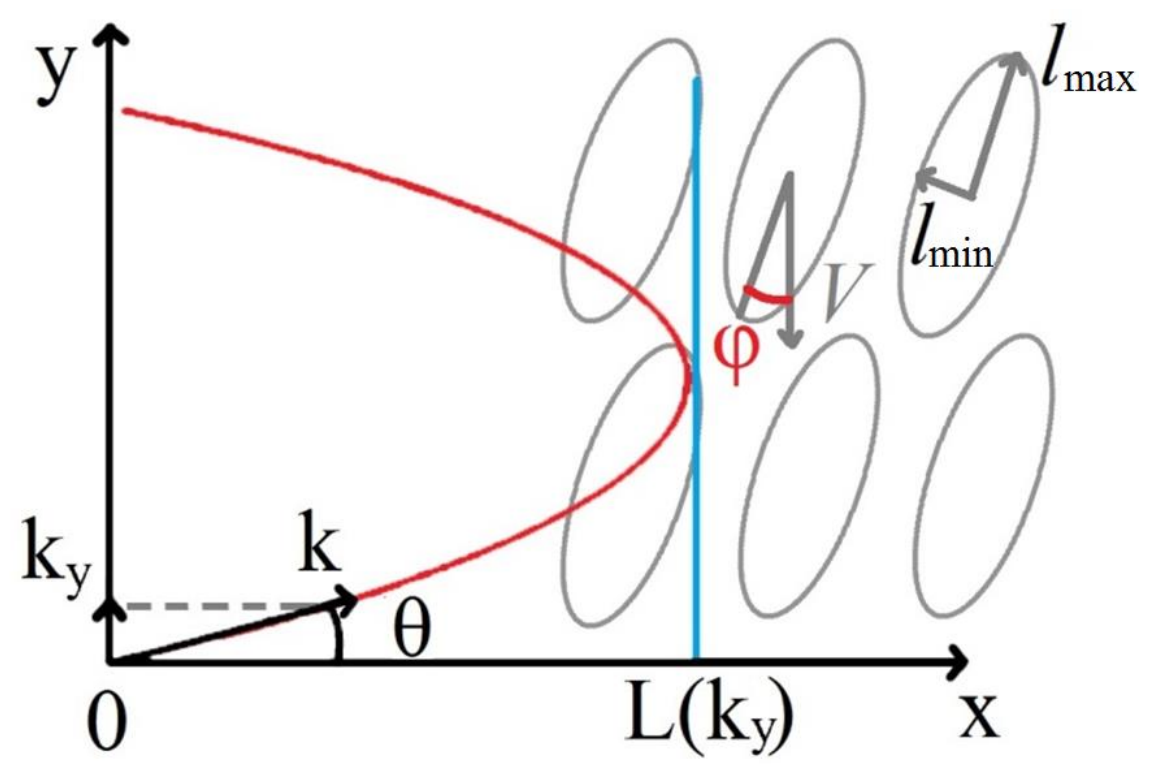

Fig.1. Simplified scheme of the theoretical model geometry defining part of variables.

In this section the goal is to outline the model used and derive general expressions for the scattered fields and a cross-correlation function measured in experiment, in much the same manner as it is done in $[5,7,11]$. The differences corresponding to the tilted turbulence effects and paraxial approximation utilized in this paper will be introduced in the next section.

The propagation of the O-mode in tokamak is described by the Helmholtz equation for the electric field component $E_{z}$ aligned with the external magnetic field:

$$
\frac{d^{2}}{d x^{2}} E_{z}(x, y)+\frac{d^{2}}{d y^{2}} E_{z}(x, y)+k^{2}(x, y) E_{z}=0
$$

The wave number $k$ for O-mode is defined as $k^{2}(x, y)=\omega^{2} / \mathrm{c}^{2}\left(1-n(x, y) / n_{c}\right)$ where $n_{c}=m_{e} \omega^{2} / 4 \pi e^{2}$ is cut-off density. That in turn allows us to relate wave number perturbation to the density perturbation: $\quad k_{0}^{2}(x)=\omega^{2} / \mathrm{c}^{2}\left(1-n_{0}(x) / n_{c}\right)=\omega^{2} / \mathrm{c}^{2}(1-x / L) ; \quad \delta k^{2}(x, y)=-\omega^{2} / \mathrm{c}^{2} \delta n(x, y) / n_{c} . \quad$ In these expressions we assume that the wave is propagating through a perturbed plasma, where density can be separated into linear poloidally uniform "background" profile $n_{0}$ and a perturbation $\delta n$ 
caused by turbulence. Here, $L$ corresponds to radial (x) coordinate of the cut-off point corresponding to the probing frequency $\omega$ and is proportional to $\omega^{2}$.

Within this paper we will restrict ourselves to considering linear scattering regime, which is adequately described within the Born approximation. Furthermore, since only the scattered signal received by antenna is of interest, reciprocity theorem [20] can be used to simplify the problem even further:

$$
A_{s}(\omega+\Omega)=\frac{i e^{2}}{4 m_{e} \omega^{2}} \omega \sqrt{P} \int_{-\infty}^{\infty} \int_{-\infty}^{\infty} \frac{\delta n_{\Omega}(x, y)}{n_{c}} E_{z}(x, y)^{2} d x d y
$$

where $A_{s}$ is the amplitude of the scattered signal received by antenna at a frequency $\omega+\Omega$ and $P$ is the probing beam power. $\delta n_{\Omega}$ corresponds to the spectral component $\Omega$ of the turbulence frequency spectrum and $E_{z}$ is normalized to a unit of power electric field. In the considered case of drift wave turbulence $\Omega<<\omega$ which is why it is going to be neglected everywhere except for the turbulence spectrum.

To obtain the signal corresponding to linear scattering it is enough to substitute expression for the unperturbed electric field (given by the solution of equation (1) when perturbation $\delta k$ is neglected) into the expression (2). Considering the background density profile to be linear, the solution of equation (1) without the density fluctuations can be expressed in terms of an integral over Airy functions corresponding to poloidal harmonics of the probing beam:

$$
\begin{aligned}
& E_{z}{ }^{0}(x, y)=\sqrt{\frac{8 \omega \alpha}{c^{2}}} \int_{-\infty}^{\infty} \frac{d k_{y}}{2 \pi} f\left(k_{y}\right) A i\left(\frac{x-L\left(k_{y}\right)}{\alpha}\right) \exp \left(i \Phi\left(k_{y}\right)-\frac{i \pi}{4}\right) ; \\
& \Phi\left(k_{y}\right)=\frac{\omega}{c} \int_{0}^{L\left(\mathrm{k}_{y}\right)} \sqrt{k_{0}^{2}(\mathrm{x})-k_{y}^{2}} d x=\frac{2}{3}\left(\frac{L\left(k_{y}\right)}{\alpha}\right)^{3 / 2} ;
\end{aligned}
$$

where $L\left(k_{y}\right)=L \times\left(1-k_{y}{ }^{2} c^{2} / \omega^{2}\right)$ is the radial coordinate at the turning point, $\alpha=\left(c^{2} L / \omega^{2}\right)^{1 / 3}$ is Airy scale and $f\left(k_{y}\right)$ - is the probing antenna pattern. We will consider a Gaussian antenna pattern given by the following expression:

$$
f\left(k_{y}\right)=\sqrt{2 \sqrt{\pi} \rho} \exp \left(-\left(k_{y}-K\right)^{2} \rho^{2} / 2\right) ;
$$

where $K=\omega \sin \theta / c$ is the probing beam poloidal wavenumber and $\theta$ is the antenna tilt angle with respect to normal probing and $\rho$ is the beam waist. Substituting the solution (3) into expression (2), we obtain an expression for the scattering signal in the linear approximation:

$$
\begin{aligned}
& A_{s}(\omega+\Omega)=\frac{2 e^{2} \alpha^{2}}{m_{e} c^{2}} \sqrt{P} \int_{-\infty}^{\infty} \frac{d \kappa}{2 \pi} \int_{-\infty}^{\infty} \frac{d q}{2 \pi} \delta n_{\Omega}(\kappa, q) \int_{-\infty}^{\infty} \frac{d k_{y}}{2 \pi} \times \\
& \times f\left(k_{y}\right) f\left(q-k_{y}\right) C\left(\kappa, q, k_{y}\right) \exp \left(i \Phi\left(k_{y}\right)+i \Phi\left(q-k_{y}\right)\right)
\end{aligned}
$$

Here, $\kappa$ and $q$ represent turbulence radial and poloidal wavenumbers respectively and $C\left(\kappa, q, k_{y}\right)$ corresponds to the scattering efficiency, definition of which can be found in [14]. Following the approach used in [7, 11] we will use the following asymptotic expression derived in [14] and corresponding to the localized Bragg backscattering: 


$$
C\left(\kappa, q, k_{y}\right)=\pi \sqrt{\frac{i \pi}{\kappa \alpha}} \exp \left(i\left(\frac{(\kappa \alpha)^{3}}{12}+\frac{\left(q-k_{y}\right)^{2} \alpha^{2}+k_{y}{ }^{2} \alpha^{2}-2 L / \alpha}{2} \kappa \alpha-\frac{\alpha^{2} q^{2}\left(2 k_{y}-q\right)}{4 \kappa \alpha}\right)\right)
$$

Substituting it into the formula (5) and performing integration over $k_{y}$ we arrive to the following expression for the scattering signal:

$$
A_{s}=\frac{e^{2} \alpha^{2}}{m_{e} c^{2}} \sqrt{\frac{i \pi}{\alpha} P} \int_{-\infty}^{\infty} \int_{-\infty}^{\infty} \frac{d \kappa d q}{2 \pi} \delta n_{\Omega}(\kappa, q) \frac{\exp \left(i \frac{(\kappa \alpha)^{3}}{12}-i L \kappa+2 i \Phi\left(\frac{q}{2}\right)+i \kappa q^{2} \frac{L c^{2}}{4 \omega^{2}}-\frac{(q-2 K)^{2} \rho^{2}}{4}\right)}{\sqrt{\kappa+i\left(\frac{\kappa L c}{\omega \rho^{2}} \frac{2-(q c / \omega)^{2}}{\sqrt{1-(q c / 2 \omega)^{2}}}+\frac{L c^{2}}{\omega^{2} \rho^{2}}\left(q^{2}-\kappa^{2}\right)\right)}} ;
$$

Before proceeding from this expression to the correlation function a few things should be mentioned. First of all, $L$ has a quadratic dependence on the probing frequency, which should be taken into account when we consider correlation of two scattering signals corresponding to different probing frequencies. Consequently, the Airy scale $\alpha$ does not depend on $\omega$.

Normalized cross-correlation function (CCF) of scattering signals separated in frequency by $\Delta \omega$ and in time by $\Delta t$ can be expressed as follows in the case of statistically stationary turbulence:

$$
C C F(\Delta \omega, \Delta t)=\frac{\left\langle\int_{-\infty}^{\infty} A_{s}(\omega+\Omega) A_{s}^{*}(\omega+\Omega+\Delta \omega) e^{i \Omega \Delta t} d \Omega\right\rangle}{\left\langle\int_{-\infty}^{\infty} A_{s}(\omega+\Omega) A_{s} *(\omega+\Omega) d \Omega\right\rangle\left\langle\int_{-\infty}^{\infty} A_{s}(\omega+\Omega+\Delta \omega) A_{s} *(\omega+\Omega+\Delta \omega) d \Omega\right\rangle} ;
$$

where brackets designate averaging over the statistical ensemble of the turbulence. Which means they are applied to $\delta n$. In this work density perturbations are assumed to be statistically homogeneous, stationary and independent, which means their two-point cross-correlation function can be determined from Wiener-Khinchin theorem:

$$
\langle\delta n(x, y, t) \delta n(x, y, t) *\rangle=\int_{-\infty}^{\infty} \int_{-\infty}^{\infty} \int_{-\infty}^{\infty} \frac{d \kappa d q d \Omega}{(2 \pi)^{3}}\left|\delta n_{\Omega}(\kappa, q)^{2}\right| \exp \left(i \kappa\left(x-x^{\prime}\right)+i q\left(y-y^{\prime}\right)+i \Omega\left(t-t^{\prime}\right)\right) ;
$$

Keeping in mind that in experimental conditions $\Delta \omega / \omega<<1$, we can now express the correlation function in terms of an integral over turbulence wavenumbers and frequency while expanding the argument of exponent in

(7) into Taylor series over $\Delta \omega / \omega$ :

$$
\begin{aligned}
& C C F(\Delta \omega, \Delta t) \approx \exp \left[-\left(\frac{\Delta \omega}{\omega} K \rho\right)^{2}\right] \frac{J(\Delta \omega, \Delta t)}{J(0,0)} \\
& J(\Delta \omega, \Delta t)=\int_{-\infty}^{\infty} \int_{-\infty}^{\infty} d \kappa d q d \Omega\left|\delta n_{\Omega}(\kappa, q)^{2}\right| \exp \left(-\frac{(q-2 K)^{2} \rho^{2}}{4}+(q-2 K) \frac{\Delta \omega}{\omega} K \rho^{2}\right) \times \\
& \times \frac{\exp \left(i 2 L \kappa \frac{\Delta \omega}{\omega}-2 i \Delta \omega \frac{d}{d \omega} \Phi\left(\frac{q}{2}\right)+i \Omega \Delta t\right)}{\sqrt{\kappa^{2}+\left(\frac{\kappa L c}{\omega \rho^{2}} \frac{2-(q c / \omega)^{2}}{\sqrt{1-(q c / 2 \omega)^{2}}}+\frac{L c^{2}}{\omega^{2} \rho^{2}}\left(q^{2}-\kappa^{2}\right)\right)^{2}}} ;
\end{aligned}
$$

This expression does not yet take into account specifics of the considered problem, and is very close to the ones obtained in $[5,7,11]$. The particularities of the tilted turbulent structure come 
from the shape of turbulent spectrum and its temporal behavior, which did not play a role in the stationary case considered in previous works.

\section{CCF in case of poloidally rotating tilted turbulence.}

To model the tilting angle measurement using RCDR, much like in $[18,19]$ we will consider turbulence with a tilted Gaussian spectrum over wavenumbers rotating uniformly in poloidal direction with velocity $V$ :

$$
\begin{aligned}
& \delta n(\kappa, q)^{2}=2 \pi \delta(\Omega-q V) l_{\min } l_{\max } \delta n_{0}{ }^{2} \times \\
& \times \exp \left(-(\kappa \cos \varphi-q \sin \varphi)^{2} l_{\min }{ }^{2} / 4-(\kappa \sin \varphi+q \cos \varphi)^{2} l_{\max }{ }^{2} / 4\right) ;
\end{aligned}
$$

Here, $\varphi$ corresponds to the turbulence tilt angle between the poloidal direction ( $y$-direction) and the stretching direction of the turbulent structure (see Fig.1), so $\varphi=0$ corresponds to the turbulence being aligned to the poloidal direction. This in turns means that for $\varphi=0 l_{\min }$ and $l_{\max }$ are radial and poloidal correlation lengths of the turbulence respectively.

To further simplify the task, we will exploit the paraxial approximation, assuming $|q-2 K|<<\omega / c$. This assumption will allow us to expand phase $\Phi$ into Taylor series over $q-2 K$ :

$$
\begin{aligned}
& \Phi=\frac{4}{3} \frac{L \omega}{c}\left(1-\left(\frac{q c}{2 \omega}\right)^{2}\right)^{3 / 2} \approx \frac{4}{3} \frac{L \omega}{c} \cos ^{3} \vartheta-2 L \cos \vartheta \sin \vartheta(q-2 K)-\frac{L c \cos 2 \vartheta}{2 \omega \cos \vartheta}(q-2 K)^{2}= \\
& =\frac{2}{3} \frac{L \omega}{c \cos \vartheta}\left(2+\sin ^{2} \vartheta+\frac{\sin ^{2} 2 \vartheta}{2}\right)-2 L \frac{\sin ^{3} \vartheta}{\cos \vartheta} q-\frac{L c \cos 2 \vartheta}{2 \omega \cos \vartheta} q^{2} ; \\
& \frac{d \Phi}{d \omega} \Delta \omega=2 \frac{L \Delta \omega}{c \cos \vartheta}\left(2+\sin ^{2} \vartheta+\frac{\sin ^{2} 2 \vartheta}{2}\right)-4 L \frac{\sin ^{3} \vartheta}{\cos \vartheta} q \frac{\Delta \omega}{\omega}-\frac{L c \cos 2 \vartheta}{2 \omega \cos \vartheta} q^{2} \frac{\Delta \omega}{\omega} ;
\end{aligned}
$$

Substituting (11) and

(12) into (10) performing integration over fluctuation natural frequency $\Omega$ and carrying constant part of $\Phi$ out of the integral:

$$
\begin{aligned}
& J(\Delta \omega, t)=l_{\min } l_{\max } \mathrm{e}^{i \Psi(\Delta \omega)} \int_{-\infty}^{\infty} \int_{-\infty}^{\infty} d \kappa d q \delta n_{0}{ }^{2} \frac{\exp \left(i \frac{\Delta \omega}{\omega}\left[2 \kappa L+\frac{L c q^{2} \cos 2 \vartheta}{2 \omega \cos \vartheta}+4 L q \frac{\sin ^{3} \vartheta}{\cos \vartheta}\right]-i q V t\right)}{\sqrt{\kappa^{2} \rho^{4}+\left(\frac{\kappa L c}{\omega} \frac{2-(q c / \omega)^{2}}{\sqrt{1-(q c / 2 \omega)^{2}}}+\frac{L c^{2}}{\omega^{2}}\left(q^{2}-\kappa^{2}\right)\right)^{2}}} \times \\
& \times \exp \left(-\frac{l_{\min }^{2}}{4} \cos ^{2} \varphi(\kappa-q \operatorname{tg} \varphi)^{2}-\frac{l_{\max }^{2}}{4} \sin ^{2} \varphi(\kappa+q \operatorname{tg} \varphi)^{2}+(q-2 K) \frac{\Delta \omega}{\omega} K \rho^{2}-\frac{(q-2 K)^{2} \rho^{2}}{2}\right)
\end{aligned}
$$

where $\Psi=2 \frac{L \Delta \omega}{c \cos \vartheta}\left(2+\sin ^{2} \vartheta+\sin ^{2} 2 \vartheta / 2\right)$ is the phase that can be excluded by calibration of the experimental scheme and for that reason will be omitted as well as constant numerical coefficients that do not influence the value of CCF in the subsequent formulae.

Integral over poloidal wavenumber $q$ can be estimated using saddle-point method: 


$$
\begin{aligned}
& J(\Delta \omega, t) \propto \int_{-\infty}^{\infty} \frac{\exp \left(i 2 L \frac{\Delta \omega}{\omega} \kappa-2 \frac{\Delta \omega}{\omega} K^{2} \rho^{2}+\frac{1}{2}\left(\frac{\Delta \omega}{\omega} K \rho\right)^{2}-\frac{l_{\min }^{2}}{4} \kappa^{2} \cos ^{2} \varphi-\frac{l_{\max }^{2}}{4} \kappa^{2} \sin ^{2} \varphi\right)}{\rho^{*}\left[\kappa^{2} \rho^{4}+\left(\frac{\kappa L c}{\omega} \frac{2-\left(q^{*} c / \omega\right)^{2}}{\sqrt{1-\left(q^{*} c / 2 \omega\right)^{2}}}+\frac{L c^{2}}{\omega^{2}}\left(q^{\left.*^{2}-\kappa^{2}\right)}\right)^{2}\right]^{1 / 2}\right.} \times \\
& \times \exp \left(+\frac{1}{2 \rho^{* 2}}\left[\frac{\kappa\left(l_{\min }^{2}-l_{\max }^{2}\right) \sin 2 \varphi}{4}-\left(2+\frac{\Delta \omega}{\omega}\right) K \rho^{2}+i V t-4 i \frac{\Delta \omega}{\omega} L \frac{\sin ^{3} \vartheta}{\cos \vartheta}\right]^{2}\right) d \kappa ;
\end{aligned}
$$

Here, $q^{*}$ and $1 / \rho^{*}$ are defined as follows:

$$
\begin{aligned}
& q^{*}=-\left[\frac{\kappa\left(l_{\max }^{2}-l_{\min }^{2}\right) \sin 2 \varphi}{4}-\left(2+\frac{\Delta \omega}{\omega}\right) K \rho^{2}+i V t-4 i \frac{\Delta \omega}{\omega} L \frac{\sin ^{3} \vartheta}{\cos \vartheta}\right] / \rho^{*^{2}} ; \\
& \rho^{*}=\sqrt{\rho^{2}+l_{\text {min }}^{2} \sin ^{2} \varphi / 2+l_{\text {max }}^{2} \cos ^{2} \varphi / 2-i \frac{\Delta x c \cos 2 \vartheta}{2 \omega \cos \vartheta}} ;
\end{aligned}
$$

$q^{*}$ corresponds to the saddle point and $1 / \rho^{*}$ is the characteristic width of the region of the main contribution into the integral over poloidal wavenumbers and for realistic parameter values $l_{\text {min }}$, $l_{\text {max }}<<\rho$ corresponds to one over probing beam waist.

For a wide enough beam the expression for saddle point simplifies becoming $q^{*}=-2 K$ which corresponds to the Bragg condition. Here $\Delta x$ is equal to the separation of cutoffs $2 L \Delta \omega / \omega$ and therefore the CCF appears to be a function of cutoff separation rather than of turning points separation given by $2 L \cos \theta^{2} \Delta \omega / \omega$. The same result was noted in previous studies $[7,10,11]$.

Next, an integral over radial wave number should be determined. Integrand in this case has a saddle point as well as two branching points in the denominator. We will start our consideration with only taking into account the saddle point, which was shown [7] to correspond to a situation when small-angle scattering off long-scale fluctuation is suppressed and the CCF reproduces turbulence correlation function. Other situations will be considered in the later sections.

\subsection{CCF at suppressed small-angle scattering.}

The saddle point $\kappa^{*}$ and the second derivative of the integrand phase in its vicinity are determined by the following expressions:

$$
\begin{aligned}
& \kappa^{*}=\frac{i \Delta x+\left(i V t-2 i \Delta x \frac{\sin ^{3} \vartheta}{\cos \vartheta}-\left(2+\frac{\Delta \omega}{\omega}\right) K \rho^{2}\right)\left(l_{\max }^{2}-l_{\min }^{2}\right) \sin 2 \varphi / 4 \rho^{*^{2}}}{\left(l_{\min }^{2} \cos ^{2} \varphi+l_{\max }^{2} \sin ^{2} \varphi\right) / 2} ; \\
& \left.\frac{d^{2} \Phi}{d \kappa^{2}}\right|_{\kappa^{*}}=-l_{\min }^{2} \cos ^{2} \varphi / 2-l_{\max }^{2} \sin ^{2} \varphi / 2+\frac{\left(l_{\max }^{2}-l_{\min }^{2}\right)^{2} \sin ^{2} 2 \varphi}{4 \rho^{2}}=-l_{c x} o^{2} / 2 ;
\end{aligned}
$$

The important thing to notice here is that anisotropy of turbulence spectrum introduces real part into the saddle point - due to the tilt of the turbulent structure the nonzero radial wavenumber is prevalent in the spectral component with nonzero poloidal wavenumber. This effect can play a role for high enough values of probing angle and stronger anisotropy.

The contribution of the saddle point to the integral takes the form: 


$$
\begin{aligned}
& J(\Delta \omega, t)^{\text {saddle }} \propto \frac{\exp \left(\frac{1}{2}\left(\frac{\Delta \omega}{\omega} K \rho\right)^{2}-2 \frac{\Delta \omega}{\omega} K^{2} \rho^{2}+\frac{1}{2 \rho^{2}}\left[-\left(2+\frac{\Delta \omega}{\omega}\right) K \rho^{2}+i V t-2 i \Delta x \frac{\sin ^{3} \vartheta}{\cos \vartheta}\right]^{2}\right)}{\rho * q_{c x}\left[\kappa^{*^{2}} \rho^{4}+\left(\frac{2 \kappa^{*} L c}{\omega}+\frac{L c^{2}}{\omega^{2}} q^{*^{2}}\right)^{2}\right]^{1 / 2}} \times \\
& \times \exp \left(\frac{\left[i \Delta x+\left(i V t-2 i \Delta x \frac{\sin ^{3} \vartheta}{\cos \vartheta}-\left(2+\frac{\Delta \omega}{\omega}\right) K \rho^{2}\right)\left(l_{\max }^{2}-l_{\min }^{2}\right) \sin 2 \varphi / 4 \rho^{*^{2}}\right]^{2}}{q_{c x}^{2}}\right) ;
\end{aligned}
$$

Since tilt angle measurement technique $[18,19]$ is based on determining the $t_{\max }$ corresponding to maximum of absolute value of $C C F(\Delta \omega, t)$, we can only consider term of exponential dependence on $t$ :

$$
\begin{aligned}
& J(t)^{\text {saddle }} \propto \exp \left(-\frac{(V t)^{2}}{2 \rho^{2}}\left(1+\frac{\left(l_{\max }^{2}-l_{\min }^{2}\right)^{2} \sin ^{2} 2 \varphi}{8 \rho^{2} q_{c x}^{o^{2}}}\right)-\frac{\Delta x V t\left(l_{\max }^{2}-l_{\min }^{2}\right) \sin 2 \varphi}{2 \rho^{2} q_{c x}^{2}}\right) \\
& \exp \left(-i \frac{V t}{\rho^{*^{2}}}\left(1+\frac{\left(l_{\max }^{2}-l_{\min }^{2}\right)^{2} \sin ^{2} 2 \varphi}{8 \rho^{2} q_{c x}^{2}}\right)\left[\left(2+\frac{\Delta \omega}{\omega}\right) K \rho^{2}+2 i \Delta x \frac{\sin ^{3} \vartheta}{\cos \vartheta}\right]\right)
\end{aligned}
$$

Next, we will consider situation when $l_{\min }, l_{\max }<<\rho$ which will allow to significantly simplify the resulting formulae. A complete formula for $t_{\max }$ without this assumption is derived in Appendix 1. The simplified version of formula

(18) takes the following form:

$$
J(t)^{\text {saddle }} \propto \exp \left(-\frac{(V t)^{2}}{2 \rho^{2}}-\frac{1}{2 \rho^{2}} \frac{\Delta x\left(l_{\max }^{2}-l_{\min }^{2}\right) \sin 2 \varphi}{l_{\min }^{2} \cos ^{2} \varphi+l_{\max }^{2} \sin ^{2} \varphi} V t-2\left(2+\frac{\Delta \omega}{\omega}\right) \frac{i K \rho^{2} V t}{\rho^{2}}+\frac{2 \Delta x}{\rho^{*^{2}}} \frac{\sin ^{3} \vartheta}{\cos \vartheta} V t\right)
$$

Substituting expression for $\rho^{*}$ into it and selecting real component of the exponent argument after neglecting smaller terms one can arrive to the following expression:

$$
J(t)^{\text {saddle }} \propto \exp \left(-\frac{(V t)^{2}}{2 \rho^{2}}-\frac{1}{2 \rho^{2}} \frac{\Delta x\left(l_{\max }^{2}-l_{\min }^{2}\right) \sin 2 \varphi}{l_{\min }^{2} \cos ^{2} \varphi+l_{\max }^{2} \sin ^{2} \varphi} V t+\frac{\Delta x}{\rho^{2}} \frac{\sin \vartheta \cos 2 \vartheta}{\cos \vartheta} V t+\frac{2 \Delta x}{\rho^{2}} \frac{\sin ^{3} \vartheta}{\cos \vartheta} V t\right)
$$

Using it, the final result for $t_{\max }$ as well as the temporal Gaussian width $\Delta_{t}$ of the CCF can be obtained:

$$
\begin{aligned}
& V t_{\max }=-\frac{1}{2} \frac{\Delta x\left(l_{\max }^{2}-l_{\min }^{2}\right) \sin 2 \varphi}{l_{\perp}^{2} \cos ^{2} \varphi+l_{\mathrm{P}}^{2} \sin ^{2} \varphi}+\Delta x t g \vartheta \\
& V \Delta_{t}=\sqrt{2} \rho
\end{aligned}
$$

The last term in the first line is a result of combining the last two terms of (20). Some remarks should be made about this result. Primary one is that while expression for $t_{\max }$ looks similar to the one obtained in the intuitive approach [19] the definition of $\Delta x$ there is different, as it is defined as the turning point separation, which in this case takes the form $\Delta x=2 L \cos ^{2} \theta \Delta \omega / \omega$. This in turn means that for higher probing angles formula (21) gives drastically different values compared to the one introduced in [19] due to a difference of a factor $\cos ^{2} \theta$. At the same time for low probing angles, as described in [7], poorly localized scattering considered in the next section starts playing a role and provides qualitatively different results. 
Another thing to note is that from the expressions (17) - (19) radial correlation length of the CCF for the case of tilted turbulence can also be estimated as $l_{c x}{ }^{2}=l_{\min }{ }^{2} \cos \varphi^{2}+l_{\max }{ }^{2} \sin \varphi^{2}$, which corresponds to a projection of the tilted turbulence spectrum on the radial coordinate.

\subsection{Influence of small-angle scattering on CCF.}

Aside from the saddle point domination described in the previous section, another situation can be considered. When the saddle point provides exponentially small input into the integral (14) compared to the input of branching point vicinity, its contribution can be neglected. We will try to estimate the CCF in this case.

To determine branching points, we will simplify denominator in expression (14) by substituting $q^{*}=-2 K$ into it. The reason for such treatment is the assumption of correlation lengths being much smaller than $\rho$ coupled with the fact that $\Delta x$ and $t$ can be estimated as having the same order of magnitude as $l_{\mathrm{cx}}$ and $\Delta_{t}$ In this case, the expression for the branching points in (14) takes form, which, under the same assumptions, naturally coincides with the one obtained in [7]:

$$
\kappa_{b r}{ }^{ \pm}=-2 L \sin ^{2} \vartheta \frac{\frac{2 L c}{\omega} \pm i \rho^{2}}{\left(\frac{2 L c}{\omega}\right)^{2}+\rho^{4}}
$$

Similarly, a criterion for the critical angle above which the small-angle scattering contribution should be negligible can be obtained. The only difference to the one introduced in [7] is due to the turbulence tilt, which causes dominance of nonzero $\kappa$ in the turbulence spectrum, as mentioned before and also complicates the expression for $l_{c x}$. The final criterion of small-angle scattering suppression in this case takes a more restricting form:

$$
\sin \vartheta^{2}>>\sin \vartheta_{*}^{2}=\frac{c}{2 \omega}\left(\frac{1}{l_{c x}}+\frac{l_{\max }^{2}-l_{\min }^{2}}{l_{c x}^{2}}|\sin 2 \varphi| \frac{\omega}{c} \sin \vartheta\right) \sqrt{1+\frac{\rho^{4} \omega^{2}}{(2 L c)^{2}}} ;
$$

The case when the branching provides a dominant contribution into the integral corresponds to the situation when the value of the integrand in $\kappa_{b r}$ is exponentially bigger than the value at $\kappa^{*}$ (which corresponds to large value of $\Delta x$, when the two points are moved far apart). In this case the main temporal dependence is determined by the value of the integrand at the branching point:

$$
J(\Delta \omega, t) \propto \operatorname{Const}(\mathrm{t}) \exp \left(\frac{1}{2 \rho^{*^{2}}}\left[\frac{\kappa_{b r}\left(l_{\max }^{2}-l_{\min }^{2}\right) \sin 2 \varphi}{4}+i V t-\left(2+\frac{\Delta \omega}{\omega}\right) K \rho^{2}-4 i \frac{\Delta \omega}{\omega} L \frac{\sin ^{3} \vartheta}{\cos \vartheta}\right]^{2}\right)
$$

This once again allows determining of the values of $t_{\max }$ and $\Delta_{t}$ :

$$
\begin{aligned}
& V t_{\max }=\frac{L \sin ^{2} \vartheta}{4 \rho^{2}} \frac{\left(l_{\max }^{2}-l_{\min }^{2}\right) \sin 2 \varphi}{\left(\frac{2 L c}{\omega \rho^{2}}\right)^{2}+1}+\Delta x t g \vartheta ; \\
& V \Delta_{t}=\sqrt{2} \rho ;
\end{aligned}
$$

Same as in the transition from (19) to (20), the tangent function in (25) arises from combining the last two terms of (24) when extracting the real part. This formula illustrates that in the case when the scattering signal is dominated by the poorly localized small-angle scattering, the dependence of $t_{\max }$ on $\Delta x$ might not provide information on the turbulence reproducing antenna 
tilt angle instead. This effect will be observed in numerical calculations in further chapters and it seriously limits applicability of this method for the measurement of the turbulence tilt angle.

This result is certainly not strict, as the temporal dependence only considered within exponent phase. Moreover, we cannot offer the limits of validity for this result without a much deeper consideration of the integration contour and the saddle point method. But we felt it was important to underline the qualitative possibility on the $t_{\max }$ not being dependent on turbulence parameters (as the first term in (25) is often negligible) and moreover, such situation will be demonstrated in calculations in the further sections.

Finally, aside from two considered cases there is an intermediate situation when the CCF is not dominated by small-angle scattering, but it still plays a significant role in determining the maximum of the CCF absolute value. In this case the interplay between small-angle scattering and back-scattering needs to be taken into account, since it is impossible to neglect either. Obtaining precise results for such a situation is problematic and its existence further complicates analysis of calculation results.

\section{Analytical results discussion}

\subsection{The model limitations}

One improtant thing to notice is that while obtained results are derived rather strictly, there are still limitations to their applicability due to the model assumptions. Such limitations are listed in this section to give the reader a better idea on the applicability of the obtained results.

First and foremost, the results of this paper are derived in Born approximation, which means they are not applicable for nonlinear regime of scattering, which corresponds to higher values of density perturbation amplitude $[8,12]$.

Another assumption made in the paper is the linear dependence of the density profile on the radial coordinate, which allowed performing explicit integration both in the scattered field (5) and in the phase expressions (3). In experimental situation, density profile is often nonlinear and moreover, X-mode probing is often used, with $k^{2}$ having dependence on both density and magnetic field resulting in a nonlinear radial profile. However, the most efficient scattering takes place in the vicinity of the cut-off and $k^{2}$ there can be approximated by a linear dependence. In such a case, the corresponding scale length should replace $L$ in the theoretical results presented here (for X-mode the expression for it is more complicated, but can be inferred from [9]). Such a method could for example be relevant for the H-mode plasma where pressure gradient in the edge area is much steeper than in the core plasma. Due to the main contribution coming from the vicinity of the cut-off deeper areas of the plasma pedestal are well described by such a substitution of gradient scale length.

The simplification of the beam waist being much larger then turbulence correlation lengths used to obtain results (21) and (25) generally holds true in RCDR experiments. Similarly, paraxial approximation used in the section 2 is usually applicable.

One more simplification was implicitly made by using the specific form (6) for scattering efficiency. While generally this form is appropriate, in some cases, for example in situations when plasma edge plays a significant role (such a case will be presented in chapter 4), a different scattering efficiency should be used. Further details on scattering efficiency and the conditions under which (6) is applicable can be found in [14]. 
Another simplification, which was not explicitly stated before is the infinite limits on the integral in (14). The actual limits correspond to $\kappa$ having to be smaller than $2 \omega / c$ for the possibility of scattering to happen. Mathematically, this corresponds to the condition of existence of stationary point in the integral over radial coordinate, when calculating $A_{s}$ (see, for example [14]). While this limitation is not relevant for isotropic turbulence, in the anisotropic case, according to formula (16), the saddle point can move out close to this limit and make it meaningful. Finally, the slab geometry considered in the paper is applicable for medium and large experimental devices. The effects of plasma curvature on RCDR results including some qualitative differences were analyzed in [10].

\subsection{Qualitative conclusions}

Aside from the aforementioned differences with the results obtained in [19] and limitation caused by small angle-scattering effects, a few other conclusions can be drawn.The main one is that, considering equation (14), one can see that aside from negligible addition to $\rho^{*}$ parameters of turbulent spectrum only participate in the formula in two combinations - one $l_{\min }{ }^{2} \cos \varphi^{2}+$ $l_{\max }^{2} \sin \varphi^{2}$ corresponding to $l_{c x}$ and the other $\left(l_{\max }{ }^{2}-l_{\min }^{2}\right) \sin 2 \varphi$. These are exactly the combinations that can be determined from $t_{\max }$ dependence on $\Delta x$ and from the CCF decay with $\Delta x$ at $t_{\max }=0$. Thus, formula (14) shows, it is not possible to resolve individual parameters $l_{\max }$, $l_{\text {min }}, \varphi$ and obtain more information on turbulence tilt from $\mathrm{CCF}$, even if a different technique is used.

Another method however, seems to be applicable - formula (17) indicates that dependence of imaginary part of CCF on $\Delta x$ also provides information on parameter $\left(l_{\max }{ }^{2}-l_{\min }{ }^{2}\right) \sin 2 \varphi$, which can be extracted. Advantage of this method is that it does not require temporal analysis of CCF. We also expect this method to be more suited for the reconstruction procedure proposed in [7] and validated in [11] to counteract the influence of small-angle scattering, as the $t_{\text {max }}$ approach did not work well with reconstruction procedure in preliminary calculations due to strong influence on numerical errors. Verification of this speculation as well as more detailed study of this alternative method will be the subject of future work. In the opposite case, when $\rho$ is comparable with the correlation lengths, additional combination of parameters is present in $\rho^{*}$ and one could theoretically obtain parameters $l_{\max }, l_{\min }, \varphi$ by fitting them into (27) and into dependence of CCF on $\Delta \mathrm{x}$ given by (17). This approach, however, was not further explored in this paper due to improbability of this situation.

\section{Numerical validation}

To validate the results obtained in the previous chapters, numerical modeling was performed using wave propagation solvers. Since full-wave calculations are demanding and results of the paper were obtained within the Born approximation, a faster approach, similar to the one described in [21] was used. Namely, the solution (3) of the unperturbed Helmholtz equation combined with turbulence spectrum (11) was used to calculate the CCF according to (2) and (8) without the need to average over random fluctuations or to calculate perturbed electric field. While simple, such an approach allowed for recovery of all the relevant physical effects in linear scattering regime. 
After calculating the CCF this way, the maximum of its absolute value for each frequency separation $\Delta \omega$ was determined and corresponding $t_{\max }$ was obtained. The dependence of $t_{\max }$ on $\Delta x$ was compared with the ones described by formula (21).

A result of applying such a procedure can be seen on the figure 2. On the figure $y_{\max }=V t_{\max }$ and $\Delta x=2 L \Delta \omega / \omega$. Parameters used for presented calculation were chosen to be relevant to experimental condition and are as follows: $L=10 \mathrm{~cm}, l_{\min }=1 \mathrm{~cm}, l_{\max }=1.5 \mathrm{~cm}, \rho=3 \mathrm{~cm}$, $\varphi=45^{\circ}, f=30 \mathrm{GHz}$.

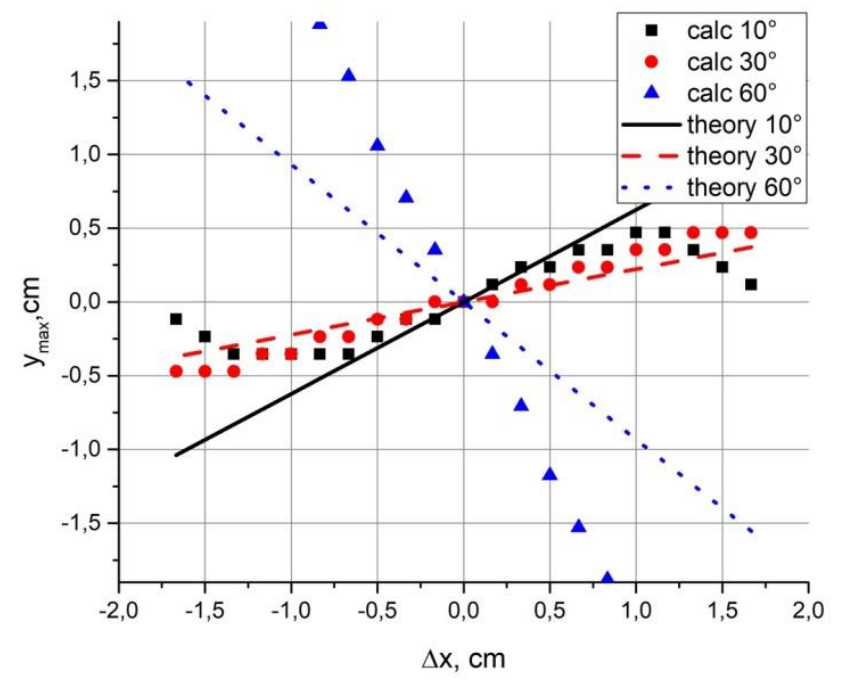

Figure 2. CCF maximum dependence on the radial separation. $L=10 \mathrm{~cm}, l_{\min }=1 \mathrm{~cm}, l_{\max }=1.5 \mathrm{~cm}, \rho=3 \mathrm{~cm}$, $\varphi=45^{\circ}, f=30 \mathrm{GHz}$. Lines correspond to formula (21), points correspond to calculation results, while different colors, line and symbol types designate different probing angles $\theta$.

It can be seen that for higher probing angle $30^{\circ}$ the agreement between theory and calculation is rather good (although not for the angle $60^{\circ}$, which we attribute to edge effects rendering scattering efficiency (6) inapplicable), whereas for low angle $10^{\circ}$ the agreement deteriorates due to small angle scattering. This is in agreement with the theoretical results, predicting that higher probing angles benefit from suppressed small-angle scattering.

A more favorable situation is shown at figure 3. In this case the input parameters are as follows: $L=4 \mathrm{~cm}, l_{\min }=0.2 \mathrm{~cm}, l_{\max }=1.4 \mathrm{~cm}, \rho=1.6 \mathrm{~cm}, \varphi=45^{\circ}, f=70 \mathrm{GHz}$. As can be seen from the figure significant disagreement can only be observed for the probing angle $10^{\circ}$. Improvement of the situation for these parameters goes in line with the small-angle scattering suppression criterion (23). 


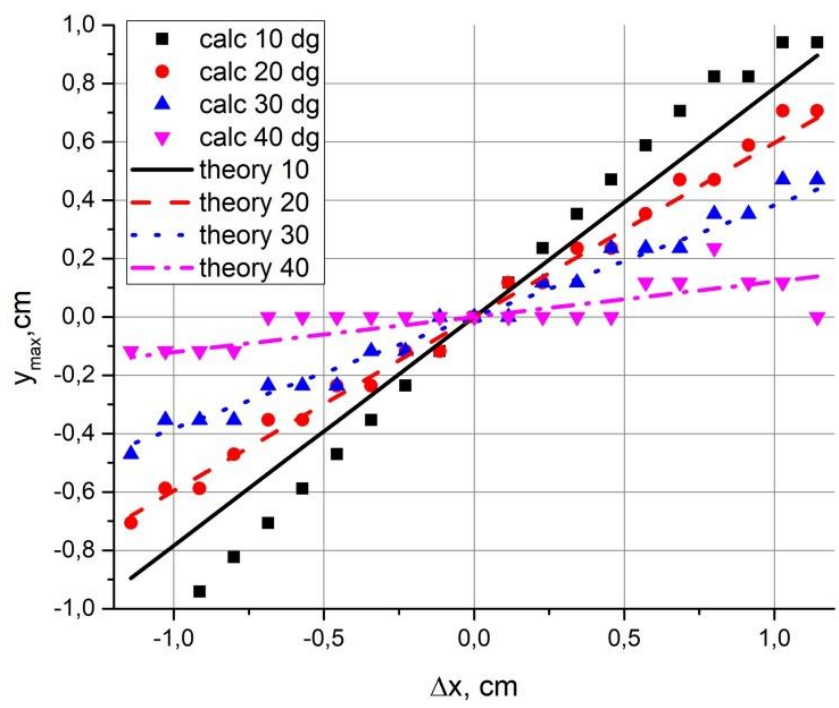

Figure 3. CCF maximum dependence on the radial separation. $L=4 \mathrm{~cm}, l_{\min }=0.2 \mathrm{~cm}, l_{\max }=1.4 \mathrm{~cm}, \rho=1.6 \mathrm{~cm}$, $\varphi=45^{\circ}, f=70 \mathrm{GHz}$. Lines correspond to formula (21), points correspond to calculation results, while different colors, line and symbol types designate different probing angles $\theta$.

To illustrate the situation when small-angle scattering dominates the scattered signal and dependence on the turbulence parameters becomes negligible, the numerical results for this case are shown on the figure 4 .

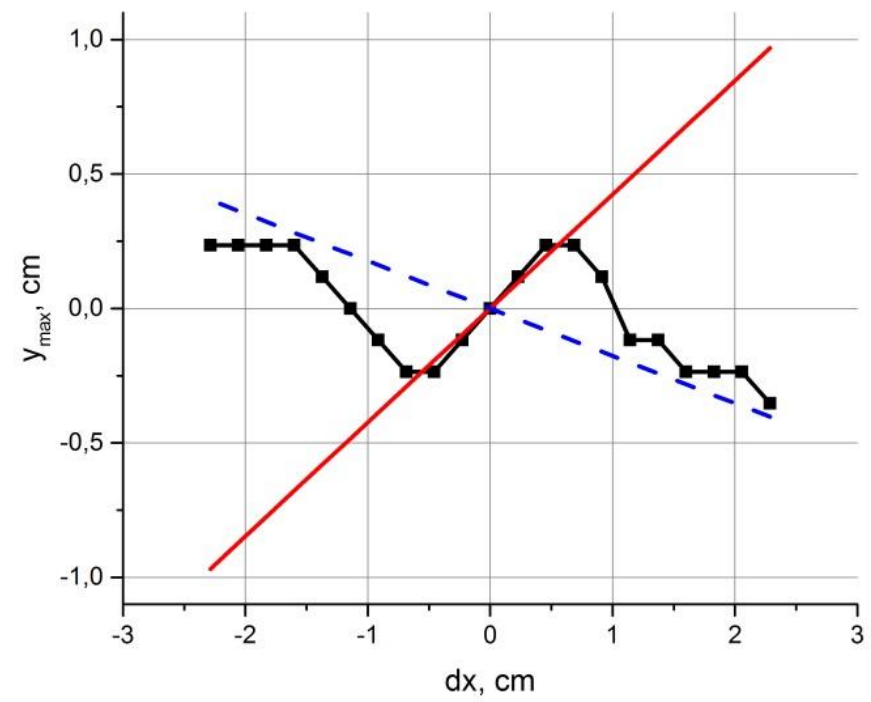

Figure 4. CCF maximum dependence on the radial separation for $L=8 \mathrm{~cm}, l_{\min }=0.5 \mathrm{~cm}, l_{\max }=1 \mathrm{~cm}, \rho=2 \mathrm{~cm}$, $\varphi=45^{\circ}, f=70 \mathrm{GHz}$. Black line with points corresponds to computation results, solid red line corresponds to theoretical formula (21) while dashed blue line corresponds to formula (25)

In agreement with the explanation given in section 2.2, for small values of $\Delta x$ the branching point does not influence integral (14) and computation results are adequately described by formula (21). As the radial separation grows, the branching and saddle points move further apart and the dependence on the turbulence parameters disappears in agreement with (25). The red line seemingly crossing origin corresponds to the fact that constant offset in formula (25) for these parameters has the value of $0.01 \mathrm{~cm}$. This corresponds to the fact that small-angle scattering 
contribution into scattering signal decays much slower with radial separation than that of the backscattering. As it can be seen from the figure 4, the influence of small angle scattering can potentially even change the sign of the linear dependence coefficient, which can be used for experimental confirmation of the theoretical predictions.

Finally, to illustrate significance of the difference in $\Delta x$ definition in this paper and in [19] figure 5 demonstrates calculated value of $y_{\max }$ together with theoretical predictions given by both formula (21) and intuitive interpretation given in [19] as a function of frequency separation. This difference being a factor $\cos ^{2} \theta$ is quite significant for larger probing angles, which seem to be more suitable as they allow suppressing small-angle scattering effects. The calculation used is the same at the one presented at figure 3 , while probing angle is $30^{\circ}$.

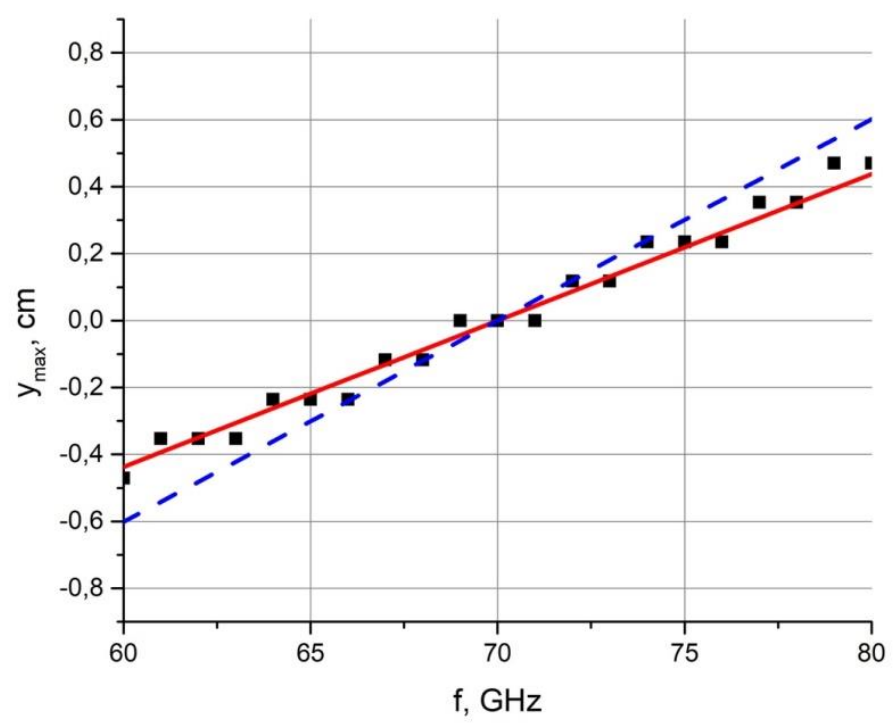

Figure 5. Dependence of $y_{\max }$ on the probing frequency. $L=4 \mathrm{~cm}, l_{\min }=0.2 \mathrm{~cm}, \quad l_{\max }=1.4 \mathrm{~cm}, \rho=1.6 \mathrm{~cm}$, $\varphi=45^{\circ}, f=70 \mathrm{GHz}$. Black symbols correspond to calculation, solid red and dashed blue lines are given by formula (21) and intuitive interpretation given in [19] respectively.

To see the impact of difference between analytical formulae we have calculated the turbulence tilt angle from calculation results according to formula (21) and to [19] neglecting $l_{\perp}$ in both cases. The results obtained were $45^{\circ}$ and $41^{\circ}$ respectively, which mean that while rigorous analytical formula provides a better estimate, the difference in the considered case is not that large.

Overall, the modeling results confirm analytical conclusions and highlight the importance of the effects caused by small angle scattering. We would also like to empathize that various effects mentioned in section 3.2, such as simplified scattering efficiency effects at higher probing angles, finite probing beam waist and saddle point $\kappa^{*}$ moving close to integration limit $2 \omega / \mathrm{c}$ for small turbulence tilt angle manifested in a number of calculations not presented in this paper. Together with small-angle scattering effects, they made the range of parameters where the calculations were in agreement with analytical prediction (21) rather limited. This observation casts doubt on the ability of the diagnostic to produce reliable quantitative experimental results and suggests that the measurements should be interpreted very carefully. 


\section{Conclusions}

In this paper we have investigated the turbulence tilt angle measurements technique within the linear approximation of analytical theory. Analytical expression for CCF was derived and key features, such as the influence of small angle scattering as well as dependence on cut-off separation rather than turning point separation of "intuitive interpretation" were pointed out. New formulae for the maximum of the CCF were derived for both a case with no small-angle scattering influence and for the one in which small-angle scattering dominates the scattered signal. A general expression, taking into account finite beam width was also obtained, demonstrating that for smaller beam waists direct interpretation is impossible.

Analytical results were validated with the use of numerical simulations employing reciprocity theorem and linear approximation. The contribution and the role of the small angle scattering that have been identified in the new formulae and the difference (which one, precise it) with "intuitive" interpretation [21] was confirmed.

\section{Acknowledgements}

The analytical investigation was performed with support of the Russian Science Foundation grant 17-12-01110, whereas numerical analysis was supported by the Ioffe Institute. This work has been carried out partially within the framework of the EUROfusion Consortium and of the French Federation for Magnetic Fusion Studies (FR-FCM) and has received funding from the Euratom research and training program 2014-2018 and 2019-2020 under grant agreement No 633053. The views and opinions expressed herein do not necessarily reflect those of the European Commission. 


\section{Appendix 1:}

If the formula (17) was to be considered for arbitrary relation between $l_{\min }, l_{\max }$ and $\rho$, one could still obtain the expression for $t_{\max }$ by finding real part of the exponent argument. The key point is to keep in mind imaginary parts of both $\rho^{*}$ and $\tilde{l}_{c x}(17)$ using them:

$$
\begin{aligned}
& A_{1}=\left|\rho^{* 2}\right|=\left(\rho^{2}+\frac{l_{\perp}^{2} \sin ^{2} \varphi}{4}+\frac{l_{\|}^{2} \cos ^{2} \varphi}{4}\right)^{2}+\left(\frac{\Delta x c \cos 2 \vartheta}{4 \omega \cos \vartheta}\right)^{2} ; \\
& R_{1}=\operatorname{Re}\left(\frac{\rho^{2}}{\rho^{*^{2}}}\right)=\frac{\rho^{2}+\frac{l_{\perp}^{2} \sin ^{2} \varphi}{4}+\frac{l_{\|}^{2} \cos ^{2} \varphi}{4}}{A_{1}} \rho^{2} ; I_{1}=\operatorname{Im}\left(\frac{\rho^{2}}{\rho^{*^{2}}}\right)=\frac{\left(\frac{\Delta x c \cos 2 \vartheta}{2 \omega \cos \vartheta}\right)}{A_{1}} \rho^{2} \\
& A_{2}=\left|\tilde{l}_{c x}^{2}\right|=\left(l_{c x}^{2}+\frac{\left(l_{\max }^{2}-l_{\min }^{2}\right)^{2} \sin ^{2} 2 \varphi}{2 \rho^{2}} R_{1}\right)^{2}+\left(\frac{\left(l_{\max }^{2}-l_{\min }^{2}\right)^{2} \sin ^{2} 2 \varphi}{2 \rho^{2}} I_{1}\right)^{2} \\
& R_{2}=\operatorname{Re}\left(\frac{l_{c x}^{2}}{\tilde{l}_{c x}^{2}}\right)=\frac{l_{c x}^{2}+\frac{\left(l_{\max }^{2}-l_{\min }^{2}\right)^{2} \sin ^{2} 2 \varphi}{2 \rho^{2}} R_{1}}{A_{2}} l_{c x}^{2} \cdot I_{2}=\operatorname{Im}\left(\frac{l_{c x}^{2}}{\tilde{l}_{c x}^{2}}\right)=\frac{-\frac{\left(l_{\max }^{2}-l_{\min }^{2}\right)^{2} \sin ^{2} 2 \varphi}{2 \rho^{2}} I_{1}}{A_{2}} l_{c x}^{2} \\
& V \tilde{t}=V t-2 \Delta x \frac{\sin ^{3} \vartheta}{\cos \vartheta} ; \Lambda=\left(l_{\max }^{2}-l_{\min }^{2}\right) \sin 2 \varphi / 4 \\
& \operatorname{Re}\left(\frac{1}{2 \rho^{*^{2}}}\left[-\left(2+\frac{\Delta \omega}{\omega}\right) K \rho^{2}+i V t-2 i \Delta x \frac{\sin ^{3} \vartheta}{\cos \vartheta}\right]^{2}\right)=-(V \tilde{t})^{2} \frac{R_{1}}{2 \rho^{2}}+2 K V \tilde{t} I_{1}+\operatorname{Const}(t) ; \\
& \operatorname{Re}\left(\frac{\left[i \Delta x+\left(i V \tilde{t}-\left(2+\frac{\Delta \omega}{\omega}\right) K \rho^{2}\right)\left(l_{\text {max }}^{2}-l_{\min }^{2}\right) \sin 2 \varphi / 4 \rho^{*^{2}}\right]^{2}}{\tilde{l}_{c x}^{2}}\right)^{2}= \\
& =\left(-\frac{(V \tilde{t})^{2}}{l_{c x}^{2}}\left(\frac{\Lambda}{\rho^{2}}\right)^{2}\left(R_{1}^{2}-I_{1}^{2}\right)+\frac{\tilde{V t}}{l_{c x}^{2}} \frac{\Lambda^{2}}{\rho^{2}} 8 K R_{1} I_{1}-\frac{2 V \tilde{t}}{l_{c x}^{2}} \frac{R_{1}}{\rho^{2}} \Lambda \Delta x\right) R_{2}+ \\
& +\left(-\frac{(V \tilde{t})^{2}}{l_{c x}^{2}}\left(\frac{\Lambda}{\rho^{2}}\right)^{2} 2 R_{1} I_{1}+\frac{V \tilde{t}}{l_{c x}^{2}} 4 K \frac{\Lambda^{2}}{\rho^{2}}\left(R_{1}^{2}-I_{1}^{2}\right)+\frac{2 V \tilde{t}}{l_{c x}^{2}} \Delta x \frac{\Lambda}{\rho^{2}} I_{1}\right) I_{2}+\operatorname{Const}(t) ;
\end{aligned}
$$

And as a result: 


$$
\begin{aligned}
& J(t)^{\text {saddle }} \propto \exp \left(-\frac{(V \tilde{t})^{2}}{\rho^{2}}\left(\frac{R_{1}}{2}+\frac{\Lambda^{2}}{\rho^{2} l_{c x}^{2}}\left(2 R_{1} I_{1} I_{2}+\left(R_{1}^{2}-I_{1}^{2}\right) R_{2}\right)\right)\right) \times \\
& \times \exp \left(2 V \tilde{t}\left(K I_{1}+\frac{2 K \Lambda^{2}}{l_{c x}^{2} \rho^{2}}\left(\left(R_{1}^{2}-I_{1}^{2}\right) I_{2}+2 R_{1} I_{1} R_{2}\right)+\frac{\Lambda \Delta x}{l_{c:}^{2} \rho^{2}}\left(R_{1} R_{2}-I_{1} I_{2}\right)\right)\right) \\
& V t_{\max }=2 \Delta x \frac{\sin ^{3} \vartheta}{\cos \vartheta}-\frac{K I_{1} \rho^{2}+\frac{2 K \Lambda^{2}}{l_{c x}^{2}}\left(\left(R_{1}^{2}-I_{1}^{2}\right) I_{2}+2 R_{1} I_{1} R_{2}\right)+\frac{\Lambda \Delta x}{l_{c x}^{2}}\left(R_{1} R_{2}-I_{1} I_{2}\right)}{2 \rho^{2} l_{c x}^{2}}\left(2 R_{1} I_{1} I_{2}+\left(R_{1}^{2}-I_{1}^{2}\right) R_{2}\right) \\
& V \Delta_{t}=\sqrt{\frac{2 \rho^{2}}{R_{1}+\frac{2 \Lambda^{2}}{\rho^{2} l_{c x}^{2}}\left(2 R_{1} I_{1} I_{2}+\left(R_{1}^{2}-I_{1}^{2}\right) R_{2}\right)}} ;
\end{aligned}
$$

Now, to tie this in with the previous results one can note that in the case of $l_{\min }, l_{\max }, \Lambda^{0.5}<<\rho$; $\Delta x \sim l_{c x}$ and $R_{1}, R_{2} \sim 1 \gg I_{1}, I_{2}$ and formula (17) coincides with Erreur! Source du renvoi introuvable.. Another result that can be inferred from this formula is that, as expected for turbulence with $\Lambda=0$ it gives $V t_{\max }=\Delta x \operatorname{tg} \theta$.

\section{References:}

[1] J. Schirmer et al Plasma Phys. Control. Fusion 491019 (2007)

[2] F. Fernández-Marina et al Nucl. Fusion 54072001 (2014)

[3] A. B. Altukhov et al Plasma Phys. Control. Fusion 58105004 (2016)

[4] D. Prisiazhniuk et al Plasma Phys. Control. Fusion 60075003 (2018)

[5] E. Z. Gusakov and A.Yu. Popov, 38th EPS Conference on Plasma Physics (2011) P4.056

[6] O. Krutkin et al Nucl. Fusion (2019)

[7] E. Z. Gusakov, M. A. Irzak and A. Yu. Popov Plasma Phys. Control. Fusion 56, 025009 (2014).

[8] E.Z. Gusakov, A.Yu. Popov, Plasma Phys. and Control. Fusion, v.44, No 11, p.2327 (2002)

[9] E. Z. Gusakov and A. Yu. Popov Plasma Phys. Control. Fusion 461393 (2004)

[10] E. Z. Gusakov and O. L. Krutkin Plasma Phys. Rep. 43 605-13 (2017)

[11] E. Z. Gusakov et al Phys. Plasmas 24, 022119 (2017)

[12] E. Blanco and T. Estrada Plasma Phys. Control. Fusion 55125006 (2013)

[13] A. B. Altukhov, A. D. Gurchenko, E. Z. Gusakov et.al. Phys. Plasmas 25, 082305 (2018)

[14] E. Z. Gusakov and M. A. Tyntarev, Fusion Eng. Des. 34-35, 501 (1997).

[15] E. Z. Gusakov and N. V. Kosolapova, Plasma Phys. Control. Fusion 53, 045012 (2011).

[16] C. Lechte et al Plasma Phys. Control. Fusion 5907500 (2017)

[17] E. Blanco and T. Estrada Plasma Phys. Control. Fusion 50095011 (2008)

[18] J. R. Pinzón et al Nucl. Fusion 59074002 (2019)

[19] J. R. Pinzón et al Plasma Phys. Control. Fusion in press (2019)

[20] A. D. Piliya, A. Yu. Popov, Plasma Phys. Control. Fusion 44, 467 (2002).

[21] A. B. Altukhov et al Physics of Plasmas 25, 082305 (2018) 